\title{
On generalized Lucas polynomials and Euler numbers
}

Ayşe Nalli and Tianping Zhang 


\title{
ON GENERALIZED LUCAS POLYNOMIALS AND EULER NUMBERS
}

\author{
AYŞE NALLI AND TIANPING ZHANG
}

Received 3 March, 2010

\begin{abstract}
In this paper we study the relationship between the generalized Lucas polynomials and the Euler numbers and give several interesting identities involving them.
\end{abstract}

2000 Mathematics Subject Classification: 11B39, 11B68, 11B83

Keywords: generalized Lucas polynomials, Euler numbers

\section{INTRODUCTION}

As usual, the Fibonacci polynomials $\left\{F_{n}(x)\right\}$ are defined by the second-order linear recurrence

$$
F_{n+2}(x)=x F_{n+1}(x)+F_{n}(x)
$$

for $n \geq 0$ and $F_{0}(x)=0, F_{1}(x)=1$.

The Lucas polynomials $\left\{L_{n}(x)\right\}$ are defined by the second-order linear recurrence

$$
L_{n+2}(x)=x L_{n+1}(x)+L_{n}(x)
$$

for $n \geq 0$ and $L_{0}(x)=2, L_{1}(x)=x$.

These polynomials are of great importance in the study of many subjects such as algebra, geometry and number theory itself. Obviously (1.1) and (1.2) have a deep relationship with Fibonacci numbers $\left\{F_{n}\right\}_{n \geq 0}$ and Lucas numbers $\left\{L_{n}\right\}_{n \geq 0}$ respectively.

Wenpeng Zhang [7] and Fengzhen Zhao [8] obtained some identities involving the Fibonacci numbers. Yuan Yi and Wenpeng Zhang [5] studied the calculation on the summation involving the Fibonacci polynomials and obtained the following

$$
\begin{aligned}
\sum_{a_{1}+\cdots+a_{k}=n} F_{a_{1}+1}(x) F_{a_{2}+1}(x) \ldots F_{a_{k}+1}(x) & = \\
& \sum_{m=0}^{\left\lfloor\frac{n}{2}\right\rfloor}\left(\begin{array}{c}
n+k-1-m \\
m
\end{array}\right)\left(\begin{array}{c}
n+k-1-2 m \\
k-1
\end{array}\right) x^{n-2 m}
\end{aligned}
$$


where $k, n$ are positive integers and the summation is over all $k$-dimension nonnegative integer coordinates $\left(a_{1}, a_{2}, \ldots, a_{k}\right)$ such that $a_{1}+a_{2}+\cdots+a_{k}=n$ where $k, n$ are any positive integers.

Tianping Zhang and Yuankui Ma [6] studied the relationship between the generalized Fibonacci polynomials and the famous Bernoulli numbers and gave several interesting identities involving them. They [4] also studied the relationships between the Chebyshev polynomials of the first kind and the Euler numbers.

In this paper we study the relationship between the generalized Lucas polynomials and the Euler numbers and give several interesting identities involving them.

\section{MAin Results}

Definition 1. Let $\left\{V_{n}(x)\right\}$ be the polynomial sequence. The generalized Lucas polynomials are defined by $V_{0}(x)=2, V_{1}(x)=P(x)$ and

$$
V_{n+2}(x)=P(x) V_{n+1}(x)+Q(x) V_{n}(x)
$$

where $P(x)$ and $Q(x)$ are polynomials of an indeterminate $x$ with real coefficients.

Definition 2 ([2]). The ordinary Euler numbers are defined by their generating function as follows

$$
F(t)=\frac{2}{e^{t}+1}=e^{E t}=\sum_{n=0}^{\infty} E_{n} \frac{t^{n}}{n !},|t|<\pi
$$

where our notation replaced $E^{m}$ by $E_{m}(m \geq 0)$, symbolically.

The first few ones are $1,-\frac{1}{2}, 0, \frac{1}{4}, \cdots$, and $E_{2 k}=0$ for $k=1,2,3, \cdots$. These numbers arise in the series expansions of trigonometric functions and are extremely important in number theory and analysis.

Theorem 1. For all positive integers $k$ and $n$ with $k \leq n$, we have the formula

$$
\begin{gathered}
\sum_{a_{1}+\cdots+a_{k}+b_{1}+\cdots+b_{k}=n} \frac{V_{a_{1}}(x)}{a_{1} !} \ldots \frac{V_{a_{k}}(x)}{a_{k} !} \frac{E_{b_{1}}}{b_{1} !} \ldots \frac{E_{b_{k}}}{b_{k} !}(\sqrt{\Delta(x)})^{b_{1}+\cdots+b_{k}} \\
=2^{k} \frac{(k \beta(x))^{n}}{n !}
\end{gathered}
$$

where $\beta(x)=\frac{P(x)-\sqrt{\Delta(x)}}{2}$ and $\Delta(x)=P^{2}(x)+4 Q(x)$.

Proof. Let $\alpha(x)=\frac{P(x)+\sqrt{\Delta(x)}}{2}$ and $\beta(x)=\frac{P(x)-\sqrt{\Delta(x)}}{2}$ denote the roots of characteristic polynomial $\lambda^{2}-P(x) \lambda-Q(x)$ of the generalized Lucas polynomial sequence $\left\{V_{n}(x)\right\}$. Then the terms of the sequence $\left\{V_{n}(x)\right\}$ can be expressed as $[1,3]$

$$
V_{n}(x)=\left(\frac{P(x)+\sqrt{\Delta(x)}}{2}\right)^{n}+\left(\frac{P(x)-\sqrt{\Delta(x)}}{2}\right)^{n} .
$$


Then we easily deduce that the generating function of $V(t, x)$ is

$$
V(t, x)=\sum_{n=0}^{\infty} V_{n}(x) \frac{t^{n}}{n !}=\sum_{n=0}^{\infty}\left(\alpha(x)^{n}+\beta(x)^{n}\right) \frac{t^{n}}{n !} .
$$

That is,

$$
V(t, x)=e^{\alpha(x) t}+e^{\beta(x) t}=e^{\beta(x) t}\left(1+e^{t \sqrt{\Delta(x)}}\right) .
$$

Therefore, we have

$$
\begin{aligned}
e^{\beta(x) t} & =\frac{V(t, x)}{1+e^{t \sqrt{\Delta(x)}}} \\
& =\frac{V(t, x)}{2} \frac{2}{1+e^{t \sqrt{\Delta(x)}}} .
\end{aligned}
$$

Then from (2.2) and (2.5) we have

$$
e^{\beta(x) t}=\frac{1}{2} \sum_{m=0}^{\infty} V_{m}(x) \frac{t^{m}}{m !} \sum_{n=0}^{\infty} E_{n} \frac{(t \sqrt{\Delta(x)})^{n}}{n !} .
$$

Note that for two absolutely convergent power series $\sum_{n=0}^{\infty} a_{n} t^{n}$ and $\sum_{n=0}^{\infty} b_{n} t^{n}$, we have

$$
\left(\sum_{n=0}^{\infty} a_{n} t^{n}\right)\left(\sum_{n=0}^{\infty} b_{n} t^{n}\right)=\sum_{n=0}^{\infty}\left(\sum_{u+v=n}^{\infty} a_{u} b_{v}\right) t^{n},
$$

so for the $k^{\text {th }}$ power of both sides of formula (2.6) we have

$$
\begin{gathered}
\text { LHS }=\left(e^{\beta(x) t}\right)^{k}=e^{k \beta(x) t}=\sum_{n=0}^{\infty} \frac{(k \beta(x))^{n}}{n !} t^{n}, \\
R H S=\sum_{n=0}^{\infty}\left(\frac{1}{2^{k}} \sum_{a_{1}+\cdots+a_{k}+b_{1}+\cdots+b_{k}=n} \frac{V_{a_{1}}(x)}{a_{1} !}\right. \\
\left.\ldots \frac{V_{a_{k}}(x)}{a_{k} !} \frac{E_{b_{1}}}{b_{1} !} \ldots \frac{E_{b_{k}}}{b_{k} !}(\sqrt{\Delta(x)})^{b_{1}+\cdots+b_{k}}\right) t^{n} .
\end{gathered}
$$

Comparing the coefficients of $t^{n}$, we obtain the following identity

$$
\begin{gathered}
\sum_{a_{1}+\cdots+a_{k}+b_{1}+\cdots+b_{k}=n} \frac{V_{a_{1}}(x)}{a_{1} !} \ldots \frac{V_{a_{k}}(x)}{a_{k} !} \frac{E_{b_{1}}}{b_{1} !} \ldots \frac{E_{b_{k}}}{b_{k} !}(\sqrt{\Delta(x)})^{b_{1}+\ldots+b_{k}} \\
=2^{k} \frac{(k \beta(x))^{n}}{n !} .
\end{gathered}
$$

If we take $P(x)=2 x$ and $Q(x)=1$ in Theorem 1, then we have 
Corollary 1. For all positive integers $k$ and $n$ with $k \leq n$, we have

$$
\begin{gathered}
\sum_{a_{1}+\cdots+a_{k}+b_{1}+\cdots+b_{k}=n} \frac{P_{a_{1}}(x)}{a_{1} !} \ldots \frac{P_{a_{k}}(x)}{a_{k} !} \frac{E_{b_{1}}}{b_{1} !} \ldots \frac{E_{b_{k}}}{b_{k} !}\left(2 \sqrt{x^{2}+1}\right)^{b_{1}+\cdots+b_{k}} \\
=2^{k} \frac{(k \beta(x))^{n}}{n !},
\end{gathered}
$$

where $\left\{P_{n}(x)\right\}$ is the Pell-Lucas Polynomial sequence, $\beta(x)=x-\sqrt{x^{2}+1}$.

If we take $P(x)=x$ and $Q(x)=1$ in Theorem 1, then we have

Corollary 2. For all positive integers $k$ and $n$ with $k \leq n$, we have

$$
\begin{gathered}
\sum_{a_{1}+\cdots+a_{k}+b_{1}+\cdots+b_{k}=n} \frac{L_{a_{1}}(x)}{a_{1} !} \ldots \frac{L_{a_{k}}(x)}{a_{k} !} \frac{E_{b_{1}}}{b_{1} !} \ldots \frac{E_{b_{k}}}{b_{k} !}\left(\sqrt{x^{2}+4}\right)^{b_{1}+\cdots+b_{k}} \\
=2^{k} \frac{\left(k \beta^{*}(x)\right)^{n}}{n !},
\end{gathered}
$$

where $\left\{L_{n}(x)\right\}$ is Lucas Polynomial sequence, $\beta^{*}(x)=\frac{x-\sqrt{x^{2}+4}}{2}$.

If $P(x)$ and $Q(x)$ in Theorem 1 are integers with $P^{2}+4 Q>0$ and $V_{n+2}=$ $P V_{n+1}+Q V_{n}$, where $V_{0}=2, V_{1}=P$, then we obtain the following

Corollary 3. For all positive integers $k$ and $n$ with $k \leq n$, we have the identity

$$
\begin{gathered}
\sum_{a_{1}+\cdots+a_{k}+b_{1}+\cdots+b_{k}=n} \frac{V_{a_{1}}}{a_{1} !} \ldots \frac{V_{a_{k}}}{a_{k} !} \frac{E_{b_{1}}}{b_{1} !} \ldots \frac{E_{b_{k}}}{b_{k} !}\left(\sqrt{P^{2}+4 Q}\right)^{b_{1}+\cdots+b_{k}} \\
=2^{k} \frac{\left(k \beta^{\bullet}(x)\right)^{n}}{n !}
\end{gathered}
$$

where $\beta^{\bullet}(x)=\frac{P-\sqrt{P^{2}+4 Q}}{2}$.

Taking $x=1$ in Corollary 2 or $P=Q=1$ in Corollary 3 , we obtain the identity

Corollary 4 ([4]). For all positive integers $k$ and $n$ with $k \leq n$, we have

$$
\sum_{a_{1}+\cdots+a_{k}+b_{1}+\cdots+b_{k}=n} \frac{L_{a_{1}}}{a_{1} !} \ldots \frac{L_{a_{k}}}{a_{k} !} \frac{E_{b_{1}}}{b_{1} !} \ldots \frac{E_{b_{k}}}{b_{k} !}(\sqrt{5})^{b_{1}+\ldots+b_{k}}=2^{k} \frac{\left(k \beta^{\prime}(1)\right)^{n}}{n !},
$$

where $\beta^{\prime}(1)=\frac{1-\sqrt{5}}{2}$.

In particular, taking $k=1,2,3$ in Corollary 4 , we easily get 
Corollary 5. [4] For all positive integers $n$, we have

$$
\sum_{a_{1}+b_{1}=n} \frac{L_{a_{1}}}{a_{1} !} \frac{E_{b_{1}}}{b_{1} !}(\sqrt{5})^{b_{1}}=2 \frac{\left(\beta^{\prime}(1)\right)^{n}}{n !} .
$$

Corollary 6. [4] For all positive integers $n \geq 2$, we have

$$
\sum_{a_{1}+a_{2}+b_{1}, b_{2}=n} \frac{L_{a_{1}}}{a_{1} !} \frac{L_{a_{2}}}{a_{2} !} \frac{E_{b_{1}}}{b_{1} !} \frac{E_{b_{2}}}{b_{2} !}(\sqrt{5})^{b_{1}+b_{2}}=2^{2} \frac{\left(2 \beta^{\prime}(1)\right)^{n}}{n !} .
$$

Corollary 7 ([4]). For all positive integers $n \geq 3$, we have

$$
\sum_{a_{1}+a_{2}+a_{3}+b_{1}+b_{2}+b_{3}=n} \frac{L_{a_{1}}}{a_{1} !} \frac{L_{a_{2}}}{a_{2} !} \frac{L_{a_{3}}}{a_{3} !} \frac{E_{b_{1}}}{b_{1} !} \frac{E_{b_{2}}}{b_{2} !} \frac{E_{b_{3}}}{b_{3} !}(\sqrt{5})^{b_{1}+b_{2}+b_{3}}=2^{3} \frac{\left(3 \beta^{\prime}(1)\right)^{n}}{n !} .
$$

\section{REFERENCES}

[1] G. H. Hardy and E. M. Wright, An introduction to the theory of numbers. Oxford: Oxford University Press, 1962.

[2] T. Kim, "On the $q$-extension of euler and genocchi numbers." J. Math. Anal. Appl., vol. 326, no. 2, pp. 1458-1465, 2007.

[3] T. Koshy, Fibonacci and Lucas numbers with applications, ser. Pure and Applied Mathematics (New York). New York: Wiley-Interscience, 2001.

[4] Y. K. Ma and T. P. Zhang, "An identity involving the first-kind chebyshev polynomials and the euler numbers," J. Ningxia Univ. Nat. Sci. Ed., vol. 27, no. 1, pp. 13-14,17, 2006.

[5] Y. Yuan and W. Zhang, "Some identities involving the fibonacci polynomials," Fibonacci Quart., vol. 40, no. 4, pp. 314-318, 2002.

[6] T. Zhang and Y. Ma, "On generalized fibonacci polynomials and bernoulli numbers," J. Integer Seq., vol. 8, no. 5, p. 6, 2005.

[7] W. Zhang, "Some identities involving the fibonacci numbers," Fibonacci Quart., vol. 35, no. 3, pp. 225-229, 1997.

[8] F. Zhao and T. Wang, "Generalizations of some identities involving the fibonacci numbers," Fibonacci Quart., vol. 39, no. 2, pp. 165-167, 2001.

Authors' addresses

Ayşe Nalli

Department of Mathematics, Selcuk University, 42031, Campus-Konya, Turkey

E-mail address: aysenalli@gmail.com

Tianping Zhang

College of Mathematics and Information Science, Shannxi Normal University, Xi' an, 710062, Shaanxi,

P. R. China

E-mail address: tpzhangesnnu.edu.cn 